\title{
Open Source Collaboration for Fostering Off-The-Shelf Components Selection
}

\author{
Claudia Ayala ${ }^{1}$, Carl-Fredrik Sørensen ${ }^{2},{\text { Reidar } \text { Conradi }^{2}, \text { Xavier Franch }}^{1}$, \\ Jingyue $\mathrm{Li}^{2}$ \\ 1 Technical University Of Catalunya (UPC), Software Department \\ Campus Nord- Omega Building. Barcelona, Spain \\ \{cayala,franch\}@1si.upc.edu \\ WWW home page: http://www.lsi.upc.es/ webgessi/index.html \\ 2 Norwegian University of Science and Technology (NTNU) \\ Department of Computer and Information Science \\ NO-7491, Trondheim, Norway \\ \{carlfrs,conradi,jingyue\}@idi.ntnu.no \\ WWW home page: http://www.idi.ntnu.no/grupper/su/
}

\begin{abstract}
The use of Off-The-Shelf software components in ComponentBased Development implies many challenges. One of them is the lack of available and well-suited data to support selection of suitable OTS components. This paper proposes a feasible and incremental way to federate and reuse the different efforts for finding, selecting, and maintaining OTS components in a structured way. This is done not only for supporting OTS components selection, but also to overcome reported problems with the integration and maintenance of component repositories. It is based on the "open source collaboration" idea to incrementally build an OTS components reuse infrastructure, enabling automatic support for OTS selection processes.
\end{abstract}

Keywords: Off-the-Shelf components (OTS), Commercial-Off-The-Shelf (COTS), Open Source Software (OSS), open source collaboration, component selection, reuse.

\section{Introduction}

The use of Off-The-Shelf software components -hereafter OTS- as part of large software systems have grown steadily [1]. Consequently, a huge amount of OTS has become accessible in the market. OTS mainly come in two major kinds: COTS (Commercial-Off-The-Shelf) owned by commercial vendors that often provide specific support [1]; and OSS (Open-Source-Software) provided by open source communities with freely accessible source code, but with no promise of specific

Please use the following format when citing this chapter:

Ayala, C., Sørensen, C.-F., Conradi, R., Franch, X., Li, J., 2007, in IFIP International Federation for Information Processing, Volume 234, Open Source Development, Adoption and Innovation, eds. J. Feller, Fitzgerald, B., Scacchi, W., Sillitti, A., (Boston: Springer), pp. 17-30. 
support [2]. Especially in the latter case, software engineering researchers and practitioners have become increasingly aware of the contribution that open source development is offering to the software industry, business, and society in general [3].

An OTS is defined as: "a software product that is publicly available at some cost or with some licensing obligations and other software projects can reuse and integrate it into their own products" [4]. The selection of OTS has been recognized as a critical process in the OTS-based development risk mitigation [5]. Regardless the specific properties of OSS and COTS components, we may consider that the high-level selection process (i.e. the practice of locating candidates, evaluating them with respect to the system requirements, and making the final choice) is essentially similar.

From our empirical studies aimed to investigate the state-of-the-practice in industrial OTS selection projects, we realized that their success is highly dependent on the quality and completeness of the data available concerning these components. Currently, these data are highly heterogeneous, since it comes from different sources, and sometimes its trustworthiness is unclear. Also, in those contexts (consultant companies, some IT departments, etc.) that select OTS periodically, the reuse of these data would improve the effectiveness of the selection processes. Heterogeneity and lack of reuse have a negative effect on the perceived risks of using OTS for integrating large industrial systems [6]. In fact, it is considered a major challenge for fostering the adoption of OTS in industrial frameworks, especially for OSS components [7]. This problem is even more evident for OSS components given their free and collaborative development nature that lacks of a structured documentation and a marketing channel behind. In this paper, we describe a feasible and incremental way to federate and reuse the actual efforts for selecting, and maintaining OTS in a structured way. We propose a Wiki-based portal based on a flexible metamodel that enables people (e.g., research groups, individuals or organizations) to work collectively in an open-source-like environment for obtaining, sharing, managing, storing, retrieving, and reusing OTS information for supporting the (re)use of OTS.

\section{State-of-the-Art and State-of-the-Practice}

\subsection{Industrial Practice}

Some empirical studies in companies using OTS show several relevant results about how they select and use such components [8]. Such studies reveal that they do not normally use any formal process for selecting components. Instead, most of them are using an experience-based and/or hands-on trial-based selection processes. In the first case, developers already have experience with some specific components or technology, and this experience is important in deciding which components to choose. In the second case, the World Wide Web is used to find executable components and a few of them are then downloaded and further evaluated. Additionally, such studies also demonstrated that OSS components are rarely modified, but used and integrated as is. Based on these empirical studies, we highlight in Table 1 the high-level activities directly related to the OTS selection 
processes once the decision to acquire OTS is made. These activities are not intended to fully describe OTS selection processes; but provide a general description with the goal of identifying the most relevant required roles. These roles are informal and implicit played by the respondents (i.e. they were not explicitly established in the actual practice). We also observe that in the case of organizations that continuously perform OTS selection processes, it is important to reuse their knowledge about the components and decisions taken. Thus, we envisage the Knowledge Keeper role, even when an explicit documentation is not formally written, but existing as tacit knowledge in the head of the involved people [8].

Table 1. Activities and Roles in OTS Selection

\begin{tabular}{|c|l|}
\hline $\begin{array}{c}\text { Activity } \\
\begin{array}{c}\text { Finding Candidates } \\
\text { OTS }\end{array}\end{array}$ & $\begin{array}{l}\text { Market Watcher }(M W) \text { explores the marketplace segments to find } \\
\text { components that may match the established requirements. }\end{array}$ \\
\hline $\begin{array}{c}\text { Evaluating OTS } \\
\text { Candidates }\end{array}$ & $\begin{array}{l}\text { Quality Engineer }(Q E) \text { measures the factors that are related to the } \\
\text { requirements in the candidate components. }\end{array}$ \\
\hline $\begin{array}{c}\text { Deciding OTS } \\
\text { Component }\end{array}$ & $\begin{array}{l}\text { Selector }(S) \text { takes the final decision based on the evaluation of the } \\
\text { candidates and also taking into account other relevant information } \\
\text { (mainly organizational). }\end{array}$ \\
\hline $\begin{array}{c}\text { Documenting the } \\
\text { Decision }\end{array}$ & $\begin{array}{l}\text { Knowledge Keeper }(K K) \text { stores and documents the produced } \\
\text { information and the decisions taken in the process for their future } \\
\text { use in forthcoming selection processes. }\end{array}$ \\
\hline
\end{tabular}

\subsection{Existing Resources for Supporting OTS Selection}

From the state-of-the-art review, we found that researchers and practitioners have been dealing with COTS components selection for a quite time and several selection methods and tools have been proposed, for instance: CARE, OTSO, PECA, PORE, etc., see [9] for a survey. Moreover, in the last years the use of OSS components has brought out extraordinary research interest and specific selecting approaches have been put forward [2],[10],[11]. However, almost all of these proposals focus their efforts on the evaluation of OTS, instead of locating components in a huge and changing marketplace.

Component location is usually supported by component repository systems. However, although reusable component repositories have been an active research area for more than a decade, they have not yet received wide success in practice, mainly because of too heavy upstart and maintainance cost; and undercritical information relevance later on [12],[13]. Moreover, a similar challenge to deal with OTS repositories have also been recognized [14].

To further investigate the problems that small and medium companies face when selecting OTS, we have recently performed an explorative survey in some Norwegian companies [15]. Our results draw that the components identification complexity in industrial settings is actually twofold:

- How to know which kind of components are available and which of them could be useful to solve a specific problem? There is an increasing need for organizing the OTS information available to achieve more efficient and reliable selection 
processes [16]. However, the effort is considerable due to the size and variability of the software market and the difficulty to collect and update information. It is thus a hard task for enterprises, particularly for small and medium ones, which can not invest enough time, money, and effort into component management to gain qualified information.

- How to find and process the information referred to those components to perform an effective evaluation? Even when COTS and OSS components information are supported by their specific vendors and open source community projects respectively, the kind of information they contain is often not detailed enough, and usually unstructured, presented in many different forms (e.g., forums, documents, etc.), very difficult to be processed for an objective evaluation [6],[7],[17].

Furthermore, from the answers of our respondents we figure out that the World Wide Web is the most used means to find candidate components (i.e., search on available catalogues or specialized search engines) followed by colleague recommendations. We also asked about the resources they usually use to locate components and information about them, as well as the perceived utility of such information for performing the different OTS selection activities.

Summarizing the answers, in Table 2, we provide an excerpt of the most mentioned resources, their key characteristics as well as the utility of their information to the roles tasks.

Table 2. Some Available Web Resources for Supporting OTS Selection

\begin{tabular}{|c|c|c|c|c|c|c|}
\hline \multirow{2}{*}{ Name } & \multirow{2}{*}{ Scope } & \multirow{2}{*}{$\begin{array}{l}\text { Components } \\
\text { Information }\end{array}$} & \multicolumn{4}{|c|}{ Support to the Roles } \\
\hline & & & MW & $\mathbf{Q E}$ & $\mathbf{S}$ & $\mathbf{K K}$ \\
\hline COTS Vendors & COTS & Non-Structured (NS) & $*$ & $*$ & $*$ & - \\
\hline OSS Project & OSS & NS & $*$ & $*$ & $*$ & - \\
\hline SourceForge.net & OSS & NS & $\sqrt{ }$ & - & - & - \\
\hline ComponentSource.com & Mainly COTS & NS & $\sqrt{ }$ & - & - & - \\
\hline Tigris.org & $\begin{array}{c}\text { OSS-Soft. } \\
\text { Engineering related }\end{array}$ & NS & $\sqrt{ }$ & $*$ & $*$ & - \\
\hline OpenCores.org & OSS-IP & NS & $\sqrt{ }$ & $*$ & - & - \\
\hline KnowledgeStorm.com & Mainly COTS & $\mathbf{N S}$ & $\sqrt{ }$ & $*$ & - & - \\
\hline CMSmatrix.org & OTS-CMS & Semi-Structured (SS) & $*$ & $*$ & - & - \\
\hline Messangingmatrix.com & OTS-Messaging & SS & $*$ & $*$ & - & - \\
\hline TheServerSide.com & Java & NS & $\sqrt{ }$ & - & - & - \\
\hline Freshmeat.net & Mainly OSS & SS & $*$ & $*$ & - & - \\
\hline Forrester.com & Broad IT Solutions & NS & $*$ & - & - & - \\
\hline Gartner.com & Broad ITSolutions & NS & * & - & - & - \\
\hline
\end{tabular}

$(\sqrt{ })$ supports the task $(*)$ deals with some issues $(-)$ does not deal with the task

In Table 3, we sum up our assessment of role-related current practices, their problems and implied challenges. The challenges can be summarized as the need of combining: Understandable Taxonomies, a Common Component Description Metamodel embracing all the informational dimensions for evaluating OTS, and a Reuse Infrastructure Support also feasible to small and medium organizations that can not invest enough time and money to manage it. We realize that though many 
efforts have been paid to deal with some of these challenges (e.g., the different web resources cited in Table 2, and methods and tools mentioned at the beginning of this section) there is no consensus of their utility. Therefore, there is a gap between such efforts and their realistic application [3],[6],[7],[18],[19],[20]. This drawback generates a barrier on adoption of OTS components in large industrial projects, since they make the selection process highly risky and expensive when applying complex evaluation criteria.

Table 3. Assessment of the role-related challenges for supporting OTS Selection

\begin{tabular}{|c|c|c|c|}
\hline Role & Current Practice & Problem & Challenge \\
\hline$M W$ & $\begin{array}{l}\text { - Proliferation of cataloguing } \\
\text { initiatives from profit and non- } \\
\text { profit organizations. } \\
\text { - Catalogues containing brief and } \\
\text { unstructured descriptions of } \\
\text { some inventoried components. } \\
\text { - Most catalogues do not have a } \\
\text { clear rationale behind. }\end{array}$ & $\begin{array}{l}\times \text { Understanding and } \\
\text { using the categoriza- } \\
\text { tions may be difficult. } \\
\times \text { Several descriptions } \\
\text { of the same component. }\end{array}$ & $\begin{array}{l}\checkmark \text { Understandable Ta- } \\
\text { xonomies [18] } \\
\checkmark \text { Common Compo- } \\
\text { nent Description Meta- } \\
\text { model [6] }\end{array}$ \\
\hline$Q E$ & $\begin{array}{l}\text { - OTS providers do not provide } \\
\text { structured and enough in- } \\
\text { formation for supporting eval- } \\
\text { uation and product quality } \\
\text { assessment. }\end{array}$ & $\begin{array}{l}\times \text { Complex discove- } \\
\text { ring and structuring of } \\
\text { critical information. }\end{array}$ & $\begin{array}{l}\checkmark \text { Component Descrip- } \\
\text { tion Metamodel embra- } \\
\text { cing quality character- } \\
\text { ristics [17] }\end{array}$ \\
\hline$S$ & $\begin{array}{l}\text { - Non-Technical information a- } \\
\text { bout the component is even } \\
\text { more difficult to be located. }\end{array}$ & $\begin{array}{l}\times \text { Hard requirements } \\
\text { negotiation. } \\
\times \text { Complex decision- } \\
\text { making process. }\end{array}$ & $\begin{array}{l}\checkmark \text { Component Descrip- } \\
\text { tion Metamodel embra- } \\
\text { cing non-technical fac- } \\
\text { tors [19] }\end{array}$ \\
\hline$K K$ & $\begin{array}{l}\text { - No support for organizations } \\
\text { (mainly small \& medium) that } \\
\text { continuously select OTS to } \\
\text { reuse their knowledge about } \\
\text { them. }\end{array}$ & $\begin{array}{l}\text { x Reuse of knowledge } \\
\text { is usually tacit, leading } \\
\text { to be lost if people are } \\
\text { replaced. }\end{array}$ & $\begin{array}{l}\checkmark \text { Reuse Infrastructure } \\
\text { Support [20] }\end{array}$ \\
\hline
\end{tabular}

\section{Proposed Approach}

To deal with the mentioned challenges as a whole, we propose the GOThIC (GoalOriented Taxonomy and reuse Infrastructure Construction) method [20]. It relies on several industrial experiences undertaken under action-research premises and grounded theory. The method is intended to guide the construction of an OTS reuse infrastructure (repository) that provides well-founded and understandable taxonomies to organize all information related to them. OTS information is structured in a Component Description Metamodel (CDM) based on the ISO/IEC 9126-1 quality standard. This has been extended to support all the informational dimensions for selecting OTS and reusing the information about them [14]. In addition, some research tools have been developed for supporting the method, i.e., the DesCOTS system (Description, evaluation, and selection of COTS components) [21]. 
From the industrial evaluation of GOThIC we found some concerns regarding its practical use: heavy upstart cost (i.e., small and medium enterprises will not be able in the general case to adopt it), and difficulty to maintain complete and up-to-date information due to the highly changing nature of the OTS components marketplace. To overcome such issues, we propose to combine the GOThIC method with the creative and productive potential of "open-source collaboration". In this way, the OTS technology users (i.e., individuals, organizations, academic researchers, industrials) can be harnessed to work as a community dedicated to incrementally build and maintain an open OTS information repository. This will ensure smooth start-up and maintenance cost, as well as highly reliable information. Details of this strategy are described in next section.

\section{Our Solution: An Open Wiki-Based portal for Sharing and Reusing OTS information}

We use the potential offered by a Wiki-based portal to put forward our strategy. A Wiki (from the Hawaiian Wikiwiki meaning "fast") is a collaboratively created and iteratively improved set of web pages [22]. It is considered a powerful knowledge management tool that enables the creation of an incrementally growing system containing the shared knowledge of multiple sources in a centralized infrastructure/repository (i.e. a database server, an application server that runs the Wiki software, and a web server that serves the pages and facilitates the web-based interaction). Thus, exploiting some particular Wiki characteristics (based on the principles described by Wagner [22]) we have designed an OTS-Wiki portal. Its main high-level goals are summarized below:

- Fostering an OTS Community and Incremental Population of Content. The OTS-Wiki provides the web-based infrastructure for enabling OTS technology users to collaborate as a community in an open-source-like environment, see Fig. 1. Thus, OTS Community users are able, and even encouraged to share knowledge (e.g., experiences, components information, and vendor comments). Therefore, the incremental population of content in the portal based on the OTS Community participation is expected. We have designed proper templates and guidelines for editing and use in order to share the information in a structured way (as demonstrated in the Wikipedia, an on-line encyclopedia implemented as a Wiki).

- Federating Actual Efforts for Locating and Selecting OTS Components. In this collaborative environment, OTS Community users are encouraged to add (as hyperlinks) and comment the helpfulness of existing web-resources for locating OTS components (as those cited in Table 2, called OTS Web-Resources in Fig. 1). This is a way of having an up-to date federated list of actual web-resources that the OTS Community users can exploit. Besides the obvious advantage of using hyperlinks for allowing users to make connections and to drill down into detailed knowledge, hyperlinks are also a potential quality assurance mechanism and relevance indicator. Pages with many links to them indicate a highly useful page. This factor fosters the OTS-Wiki portal to act as a meta-portal for 
promoting the progressive homogenization (complying with CDM) of the information contained in different OTS web resources. This is because such resources have an interest of being perceived as highly useful by the OTS Community users.

- Enabling Systematic Support for Selecting and Evaluating OTS Components. Having structured OTS information enables systematic support for evaluating and choosing components. We are integrating the DesCOTS system into the OTS-Wiki [21], as stated in Fig.1. It includes a set of tools that interoperate to support the whole OTS selection process: the Quality Model Tool allows defining quality models; the OTS Evaluation Tool allows evaluating components; the OTS Selection Tool allows defining requirements that drive the OTS component selection; and the Taxonomy Tool allows organizing OTS domains as a taxonomy supporting reuse of quality models. Nevertheless, some other existing or new tools can be developed or designed for using the structured OTS component information from the OTS-Wiki portal.

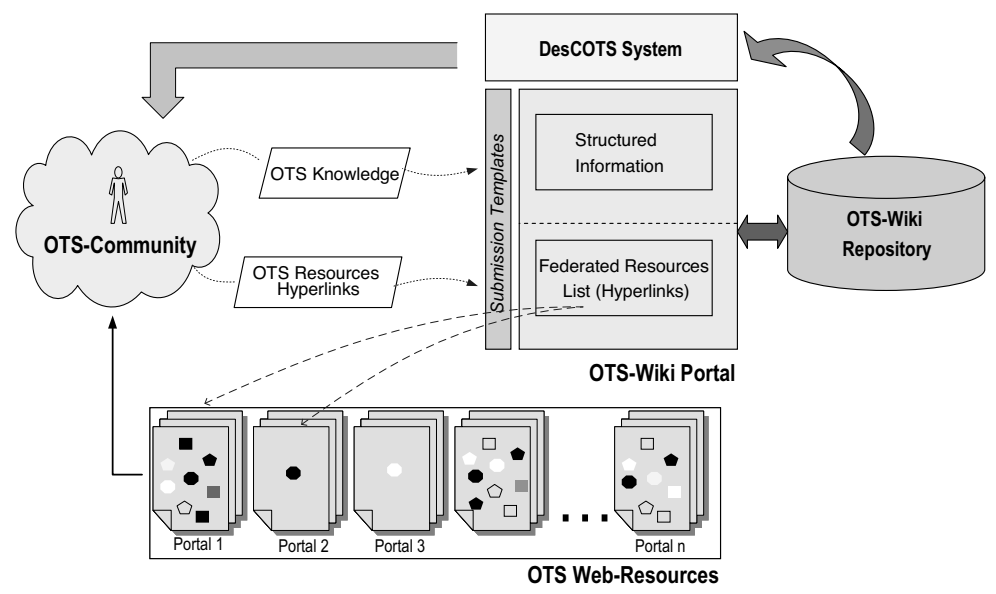

Fig. 1 OTS-Wiki Portal Main Interactions

In this scenario, any OTS Community user can use the OTS-Wiki portal as a meta-portal for providing support to: a) Locating OTS and information about them; b) Recording component information in a structured way; c) Maintaining and reusing such information; d) Getting tool support for performing selection processes. The structure of the information in the repository showed in Fig. 1 is detailed in next subsection.

\subsection{Conceptual Model of the OTS-Wiki Portal Repository}

Following the GOThIC method approach, the OTS-Wiki portal information is arranged as a goal-oriented taxonomy (composed of Market Segments and Categories) into the OTS-Wiki repository, as shown in Fig. 2. Taxonomy nodes have a generic CDM. The CDM is used as a template that can be instantiated with component information. For simplification purposes, we are not distinguishing at the moment versions of components; two different versions are treated as two different 
products. For each component, the DesCOTS system provides systematic support for OTS selection. This structure allows browsing the taxonomy and finding specific information [20].

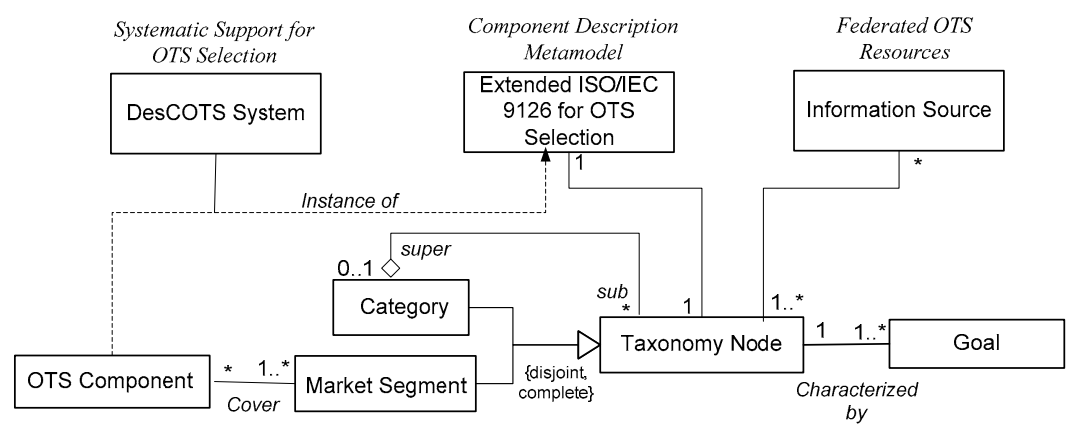

Fig. 2 Conceptual model for OTS-Wiki Portal Repository

\subsubsection{Component Description Metamodel}

The Component Description Metamodel (CDM) is based on the ISO-IEC 9126-1 software quality standard, and extended for covering all the informational dimensions needed for evaluating OTS components (functionality, quality of service, interoperability, non-technical factors and concepts definition) [14]. This structure has demonstrated to be useful for reusing information and helping the elicitation and (re)negotiation of requirements, making easier the identification of mismatches among components characteristics and the requirements in specific OTS selection processes [19]. Fig. 3 shows an excerpt of the structure of the CDM.

\begin{tabular}{|c|c|}
\hline Characteristics & Subcharacteristics \\
\hline \multirow{7}{*}{ Functionality } & Suitability \\
\hline & Suitability of Services \\
\hline & Suitability of Data \\
\hline & Accuracy \\
\hline & Interoperability \\
\hline & Security \\
\hline & F. Compliance \\
\hline Reliability & $\ldots$ \\
\hline \multirow{3}{*}{ Usability } & Understandability \\
\hline & Semantic Understandability \\
\hline & Lexical Understandability \\
\hline Efficiency & $\ldots$ \\
\hline Maintainability & $\ldots$ \\
\hline Portability & $\ldots$ \\
\hline \multicolumn{2}{|l|}{$\begin{array}{l}\text { Extended } \\
\text { Characteristics }\end{array}$} \\
\hline \multirow{4}{*}{ Supplier } & Organizational Structure \\
\hline & Positioning and Strength \\
\hline & $\ldots$ \\
\hline & Licensing Schema \\
\hline \multirow{2}{*}{ ذे Cost } & Licensing Costs \\
\hline & $\ldots$ \\
\hline \multirow{3}{*}{ Product } & Platform Cost \\
\hline & Implementation Cost \\
\hline & $\ldots$ \\
\hline
\end{tabular}

Fig. 3 Excerpt of the Component Description Metamodel 


\section{OTS-Wiki Portal Functionality Overview}

In this section, we will provide some goal-based scenario excerpts of the OTS-Wiki prototype we have implemented in order to make explicit diverse mechanisms for reaching the high-level goals stated in section 4.

Fig. 4 associates the main scenarios to reach the OTS-Wiki portal high-level goals. From scenario 4a we realize that the OTS-Wiki portal has been designed as open and freely accessible in order to enable the OTS Community in an open sourcelike environment. Scenarios $4 \mathrm{~b}, 4 \mathrm{c}$ and $4 \mathrm{~d}$ show the refinement of the high-level goals explained in section 4 into other specific sub-goals or functionalities.

\begin{tabular}{|c|c|}
\hline Goal: & Fostering OTS Community \\
\hline Description & $\begin{array}{l}\text { OTS Technology users are encouraged to work as a high performance } \\
\text { team for reusing and sharing OTS Components Information in an Open } \\
\text { and Freely accessible OTS-Wiki Portal. }\end{array}$ \\
\hline Related goal(s) & $\begin{array}{l}\text { 1.-Incremental Population of Content } \\
\text { 2.-Federation of OTS Resources } \\
\text { 3.-Enabled Systematic Support for OTS Selection }\end{array}$ \\
\hline PostCondition(s) & Progressive Foundation of OTS Community \\
\hline & $4 a)$ \\
\hline Goal: & Incremental Population of Content \\
\hline Description & $\begin{array}{l}\text { Users are encouraged to publish and share content they considered helpful } \\
\text { to the OTS Community. }\end{array}$ \\
\hline Related goal(s) & $\begin{array}{l}\text { 1.-Submit OTS Component Information } \\
\text { 2.-Enabled Active Communication } \\
\text { 3.-New Functionality Requested to the Community } \\
\text { 4.-Enabled a Glossary Construction } \\
\text { 5.-User Profiles to Personalize the Information } \\
\text {.- }\end{array}$ \\
\hline PostCondition(s) & Incremental growth of the OTS-Wiki portal content \\
\hline & $4 b)$ \\
\hline Goal: & Federation of OTS Resources in OTS-Wiki \\
\hline Description & $\begin{array}{l}\text { Users are encouraged to publish content that they consider may be helpful } \\
\text { to the Community. }\end{array}$ \\
\hline \multirow{3}{*}{ Related goal $(s)$} & $\begin{array}{l}\text { 1. - User Introduces a new OTS-Web Resource Hyperlink to the Federated } \\
\text { OTS Resources List by means of a template. } \\
\text { 1.1. - System Records the hyperlink in the OTS-Wiki Repository. }\end{array}$ \\
\hline & $\begin{array}{l}\text { 2. - User Introduces a File } \\
\text { 2.1. - Resource is uploaded to the OTS-Wiki Respository. }\end{array}$ \\
\hline & $\begin{array}{l}\text { 3. - User Provides a non-web reference } \\
\text { 3.1. - Reference is Recorded in the OTS-Wiki Repository }\end{array}$ \\
\hline \multirow[t]{2}{*}{ PostCondition(s) } & Incremental growth of the federated resources. \\
\hline & $4 c)$ \\
\hline Goal: & Enabled Systematic Support for Selection Process \\
\hline Description & $\begin{array}{l}\text { Tools are provided to support the OTS selection activities automatically, } \\
\text { using the standardized data from the repository. It is actually based on the } \\
\text { DesCOTS functionality. }\end{array}$ \\
\hline Related goal(s) & $\begin{array}{l}\text { 1.- User Requests automatic support for stating requirements } \\
\text { 2.- User Requests automatic support for matching requirements with } \\
\text { components. }\end{array}$ \\
\hline PostCondition(s) & $\begin{array}{l}\text { User is Supported to perform and document his or her selection process. } \\
\text { System Learns from each selection case (i.e. non-chosen components are } \\
\text { recorded for being shown - by analogy- to later searches) }\end{array}$ \\
\hline
\end{tabular}

Fig. 4 Goal-based Scenarios designed to reach the OTS-Wiki High-Level goals 
Fig. 5 shows some of the specific scenarios sub-goals:

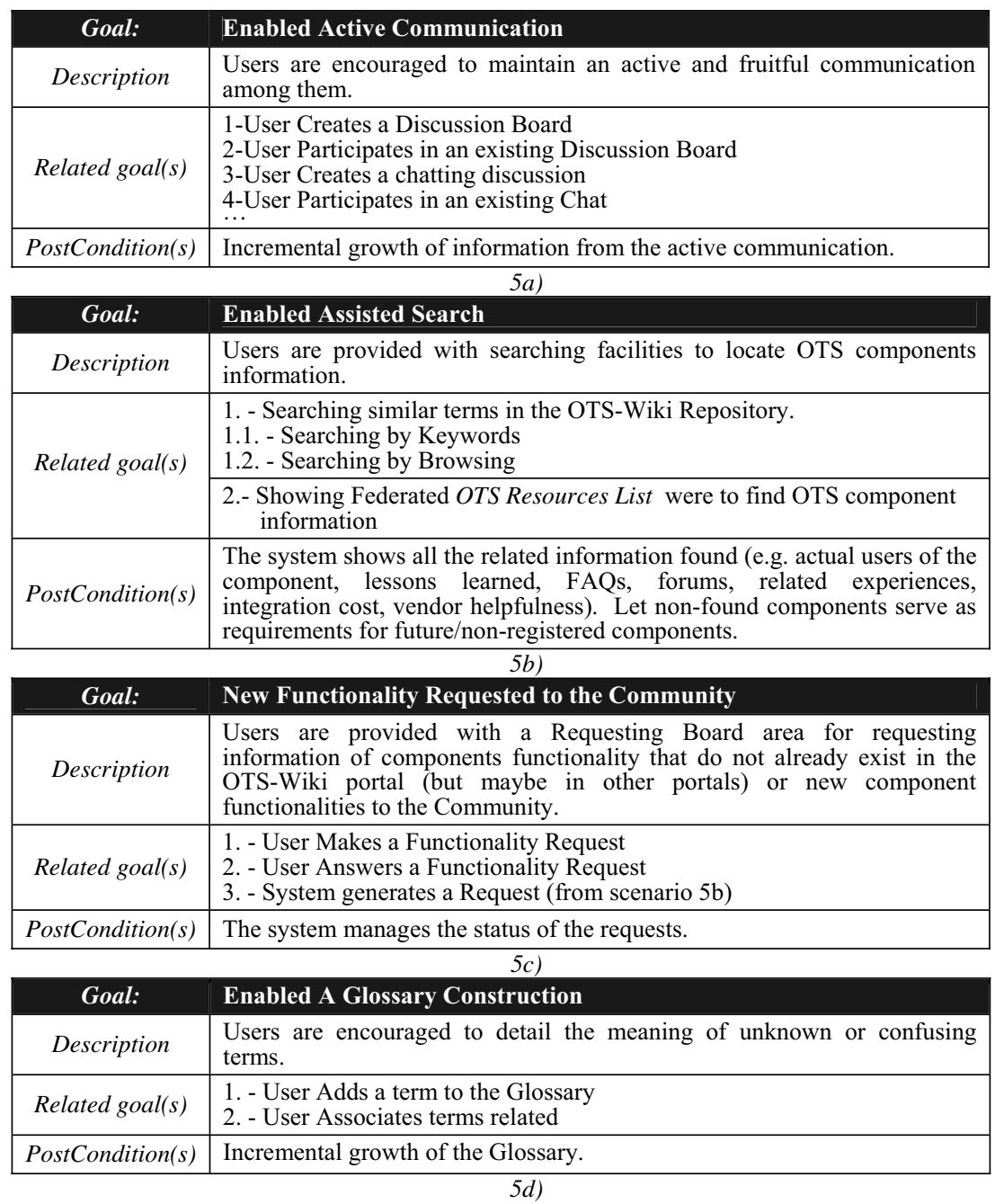

Fig. 5 Scenario excerpts to enable functionalities addressed to reach High-level OTS-Wiki Portal goals

- Fig. 5a. Enabled Active Communication: diverse mechanisms (e.g. discussion boards, chat, distribution list, etc.) are provided to enable active communication among community users.

- $\quad$ Fig 5b. Enabled Assisted Search: searching in the OTS-Wiki portal may be performed by keyword or by taxonomy navigation. The taxonomy navigation we propose (already implemented in the DesCOTS system [21]) helps users to analyze their OTS selection problem and finding their suitable market segment by navigating through a hierarchical search tree, ruling out irrelevant nodes through a question-and-answer dialog. If the information requested does not 
already exist in the OTS-Wiki repository, the system shows the Federated OTS Resources List providing hyperlinks to different resources where the information could be found; and generates a Functionality Request (Scenario 5c).

- Fig. 5c. New Functionality Requested to the Community: users are able to request and discuss component functionality not found in the OTS-Wiki portal, or with no actual implementation (those for which information was found neither in the OTS-Wiki nor in any other portal). This could result in a competition among OSS communities and COTS providers to make such components, or even encouraging the creation of new OSS communities for supporting such functionality.

- $\quad$ Fig. 5d Enabled Glossary Construction: detailing the meaning of unknown or confusing terms is important because it is common in the OTS context that the same concept may be denoted by different names in different products or even worse, the same term may denote different concepts in different products. Therefore, main concepts should be clarified via explanation pages that comprise a Glossary. This glossary also serves to provide semantic relationships among concepts via hyperlinks.

Finally, in Fig. 6 we provide a snapshot of the actual OTS-Wiki prototype and relate its functionality with the scenarios described above. Some others functionalities to be incorporated are: to provide user profiles to personalize the information to the different roles needs, and case-base reasoning support for improving the searching processes and selection of multiple components.

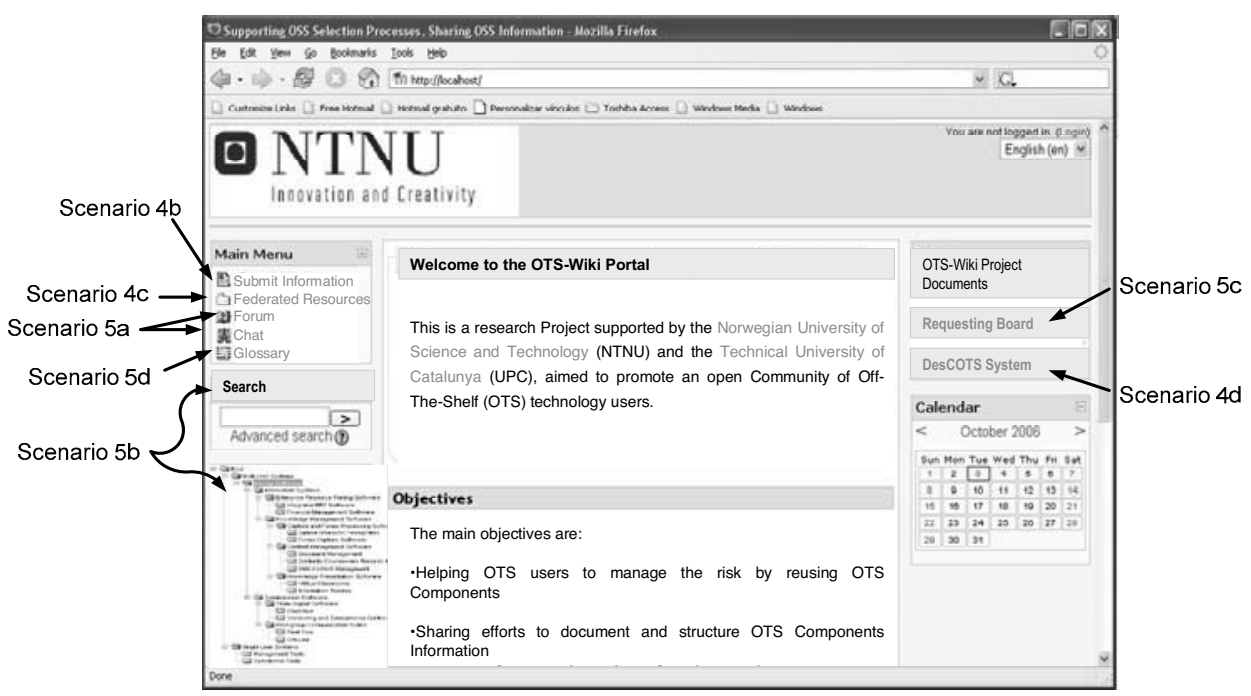

Fig. 6 OTS-Wiki Portal functionalities related with described Scenarios 


\section{Ongoing Work}

So far, we have an OTS-Wiki portal prototype combining Web-portal and Wiki technologies. It is expected to be fully operational before May 2007. Our next shortterm goal is to populate the OTS-wiki repository with some broadly used OTS components as a way to give momentum to our approach. Thus, we are taking as a base the semi-structured components information from CMSmatrix (cited in Table 2), that comprises components related with the Content Management System market segment, in order to be transformed into our proposed CDM.

On the other hand, some informational quality concerns have being discussed as user control and information ownership to provide high-quality information.

\section{Future Work}

Our intended main goal is to empirically study how the GOThIC method addresses the different issues related to OTS component selection and evaluation, as well as the effects and results of this kind of open-source-like collaboration concept for dealing with OTS selection challenges, and the problems reported with the use of repositories [12],[13]. Some metrics intended to be used are: support perceived by users, ability to enable the OTS Community, ability to promote homogenization, promotion of OSS communities, information reuse, etc. Moreover, given the social computing nature of our proposal, its functionality is going to be incrementally improved depending on the OTS Community trends and needs.

\section{Conclusion}

The proposal presented here is a feasible and incremental way of dealing with the drawbacks of OTS selection processes as well as the problems reported with the use of repositories. It is done combining the GOThIC method and the "open-source collaboration" approach in a social computing environment:

- It represents a feasible support to improve OTS selection, integration and maintenance processes as well as knowledge reuse, mainly in small and medium companies that are not able to invest enough money and time to manage a repository themselves (smooth start up and maintenance cost).

- The combination of ease and speed of publishing contents, together with the ability of engaging the potential OTS Community into the structured knowledge creation process, enables $O T S$-Wiki to become a quality platform for very large and up-to-date OTS knowledge repositories that acts as a Meta-portal for structuring the OTS unstructured information contained in other portals (this is best illustrated by the Wikipedia).

- It allows the incremental growth of a component base, where each component is linked to a community of interested users. 
- It fosters the (re)use of OTS components and promotes communities to address requirements with no actual implementation.

- $\quad$ The OTS Community interaction may address not only the challenges mentioned in section 3, but also the actual research efforts into the real needs and trends of the OTS Community.

\section{Acknowledgements}

This work has been partially supported by the Spanish MEC TIN2004-07461-C0201 project and the Norwegian COSI (Co-development and using inner \& Open source in Software Intensive products) project. C. Ayala's work has been partially supported by the Mexican Council of Science and Technology (CONACyT) and the Agència de Gestió d'Ajuts Universitaris i de Recerca (European Social Fund).

\section{References}

1. Brownsword, L., Oberndorf, T., Sledge C.A. "Developing New Processes for COTSBased Systems" IEEE Software, Vol. 17, No. 4; July-August 2000. pp.48-55.

2. Madanmohan, T.R., De, R. "Open Source Reuse in Commercial Firms" IEEE Software 21(6). 62-69.

3. Ankolekar, A., Herbsleb, J., Sycara, K. "Addressing Challenges to Open Source Collaboration with Semantic Web". In proceedings of $3^{\text {rd }}$ Workshop on Open Source Software Engineering, the 25th International Conference on Software Engineering (ICSE). 2003. Portland, Oregon, USA, pp 9-14.

4. Torchiano, M., Morisio, M. Overlooked Aspects of COTS-Based Development. IEEE Software, March/April 2004, pp 88-93.

5. Vitharana, P., Zahedi, F., Jain, H. "Knowledge-Based Repository Scheme for Storing and Retrieving Business Components: A Theoretical Design and Empirical Analysis". IEEE Transactions on Software Engineering. Vol. 29(7), 2003, pp 649-664.

6. Réquilé-Romanczuk, A., Cechich, A., Dourgnon-Hanoune, A., Mielnik, J.C., "Towards a Knowledge-based Framework for COTS components Identification" ICSE-MPEC05, ACM Press, 2005; pp 1-4.

7. Simmons, G.L., Dillon, T.S. Towards an Ontology for Open Source Software Development. In IFIP International Federation for Information Processing, Volume 203, Open Source Systems, eds. Damiani, E., Fitzgeralg, B., Scacchi, W., Scotto, M., Succi, G., (Boston:Springer), pp 65-75.

8. Li, J. Process improvement and risk management in Off-the-Shelf Component-based development. PhD Thesis 2006. Norwegian University of Science and Technology (NTNU). ISBN 82-471-7920-2. 289. http://www.idi.ntnu.no/grupper/su/publ/phd/liphdthesis-22jun06.pdf

9. Ruhe, G. "Intelligent Support for Selection of COTS Products" Proceedings Web Databases and Web Services 2002. LNCS 2593, pp. 34-45.

10. Wheeler, D.A.: How to Evaluate Open Source Software / Free Software (OSS/FS) programs. URL http://www.dwheeler.com/oss_fs_eval.html.

11. van der Berg, K. "Finding Open Options". Master Degree Thesis. Tilburg University. 2005.

12. Morisio, M., Ezran, M., and C. Tully, "Success and Failure Factors in Software Reuse," IEEE Trans. Software Eng., vol. 28, no. 4, 2002. pp. 340-357. 
13. Poulin, J. S. Populating Software Repositories: Incentives and Domain Specific Software. J Systems and Software 1995. Elsevier, pp 187-199.

14. Ayala, C., Franch, X. "Domain Analysis for Supporting Commercial Off-The-Shelf Components Selection" 25th International Conference on Conceptual Modeling (ER 2006). Tucson, Arizona, USA. November 2006. LNCS 4215, pp 354-370.

15. Gerea, M. "Selection and Evaluation of Open Source Components" Department of Computer and Information Science. Norwegian University of Science and Technology (NTNU). $\quad$ http://www.idi.ntnu.no/grupper/su/fordypningsprosjekt-2006/gereafordyp06.pdf

16. Ayala, C., Franch, X. "Transforming Software Package Classification Hierarchies into Goal-Based Taxonomies". In Proceedings of the 16th Database and Expert Systems Applications Conference (DEXA), LNCS 3588, 2005. pp 665-675.

17. Bertoa, M.F., Troya, J.M., Vallecillo, A. "A Survey on the Quality Information Provided by Software Component Vendors". In Proceedings of the 7th ECOOP Workshop on Quantitative Approaches in Object-Oriented Software Engineering (QAOOSE), 2003, pp 25-30.

18. Cechich, A., Réquilé-Romanczuk, A., Aguirre, J., Luzuriaga, J.M. "Trends on COTS Component Identification and Retrieval" In Proceedings of 5th International Conference on COTS-Based Software Systems (ICCBSS), IEEE Computer Society, 2006, pp 90-99.

19. Carvallo, J.P., Franch, X. "Extending the ISO/IEC 9126-1 Quality Model with NonTechnical Factors for COTS Components Selection" In Proceedings on the Workshop on Software Quality (WOSQ'06). IEEE Computer Society. 2006, pp. 9-14

20. Ayala, C., Franch, X. "A Goal-Oriented Strategy for Supporting Commercial Off-TheShelf Components Selection" 9th International Conference on Software Reuse (ICSR). June 2006. LNCS 4039, pp 13-24.

21. Grau, G., Carvallo, J.P., Franch, X., Quer, C., "DesCOTS: A Software System for Selecting COTS Components" In Proceedings of the 30th EUROMICRO Conference (EUROMICRO'04). IEEE Computer Society, pp 118-126.

22. Wagner, C. "Wiki: A Technology for Conversational Knowledge Management and Group Collaboration". Communications of the Association for Information Systems Vol.13, 2004. 265-289. 